\title{
Fuzzy Mathematical Model Based on Venture Investment
}

\author{
Zhaohai WANG \\ Department of mathematics and statistics, Ankang University, Ankang, Shanxi, 725000, China \\ akwzh@163.com
}

\begin{abstract}
The measurement of the investment decision is studied by applying the fuzzy analysis method, through fuzzy number arithmetic and sorting method, gives the fuzzy analysis method of net present value (rate) under the condition of risk investment decision, thus solves the risk investment decision problem in a more scientific and flexible thinking methods, it is believed that this method will get higher practical value in analysis of risk investment decision, through further improving and perfecting this method, provide more scientific decision basis to investors.
\end{abstract}

Index Terms - risk, Investment, Plan, Fuzzy, Decision making

\section{Introduction}

General measure of the investment decision, is to measure and calculate the measurable factors in investment activities, and reflect the impression of the investment plan in the face of concrete data. But, on the one hand, due to investors' limited rationality, knowledge structure of himself, personal preferences and other reasons, the accurate understanding of investment has certain limitations; Existing information and data, on the other hand, are inadequate and incomplete, and objective environmental conditions are variable, all those for the happening of the investment event, investors are difficult to make very accurate predictions.[1] [2] So, usually, the uncertainty of investment scheme is a kind of objective existence, which is the existence of the investment risk is an objective phenomenon, the investment ideas which the uncertainty factors are not included in it is blind, it also exists many instances in the real situation. In view of the uncertainty factors to consider in investment decision is necessary. [3] [6] The traditional method Dealing with uncertainty decision problems is usually a single stochastic model, which deals under the premise that assuming uncertainty event in decision-making event, regardless of its nature and form of expression is the result of some random factors. But with the emergence and development of fuzzy set theory, there is more awareness that equate uncertainty with random approach, it has a big limitation, sometimes even is unrealistic. [4] [5]

Based on this, for the uncertainty factors, this paper uses the method of fuzzy analysis, to deal with the risk value of investment. Which gives investment decision makers a scientific basis in the case of uncertainty for scheme selection. In this paper, on the basis of above analysis, through operation of analysis of the fuzzy, obtained the fuzzy representation of evaluation variable, finally based on sorting theory of the fuzzy set, presents the fuzzy analysis method of the net present value for a risk investment decision-making under the condition of risk, and through specific examples to prove the feasibility and effectiveness of the method. [7]

\section{Preliminary Knowledge}

Definition 2.1 For the fuzzy set $\tilde{A}$ on theory domain

(1) $\tilde{A}$ is a regular (normal), if and only if $\sup \mu_{\sim}(x)=1, \forall x \in X$;

(2) $\tilde{A}$ is convex, if and only if $\forall x_{1}, x_{2} \in X, \lambda \in[0,1]$,

$\mu_{\sim}\left(\lambda x_{1}+(1-\lambda) x_{2}\right) \geq \min \left\{\mu_{\tilde{A}}\left(x_{1}\right), \mu_{\tilde{A}}\left(x_{2}\right)\right\}$

Definition 2.2 a fuzzy number $\tilde{N}$ is defined on formal, convex fuzzy set in real Numbers field $\mathrm{R}$, and satisfy the following conditions:

(1) there exists the only point $x \in R$, has the membership degree $\mu_{\tilde{N}}\left(x_{0}\right)=1$ ( $x_{0}$ is called the average value of $\tilde{N}$ );

(2) the membership function $\mu_{\sim}(x)$ is left and right continuous.

The general expressions of fuzzy number $\tilde{N}$ can be written as $\mu_{\tilde{N}}(x)=\left\{\begin{array}{l}L(x), 1 \leq x \leq m, \\ R(x), m \leq x \leq r\end{array}\right.$, in which $L(x)$ is an increasing function, right continuous, and $0 \leq L(x) \leq 1$; $R(x)$ is a reduction function, left continuous, and $0 \leq R(x) \leq 1$. If $L(x)$ and $R(x)$

are all linear function, $\tilde{N}$ is called a triangular fuzzy number, short for $\tilde{N}=(1, m, r)$.

Definition 2.3 Let $f: R \rightarrow[0,1]$ be a mapping, if $f$ satisfies the following conditions:

(1) $f(x)=f(-x)$; (2) $f(0)=1$; (3) $f(x)$ is monotone decreasing in interval $[0,+\infty]$.

Then $f(x)$ is called the benchmark function of a fuzzy number.

Definition 2.4 Let $L(x)$ and $R(x)$ be left and right benchmark functions of the fuzzy number respectively, if 


$$
\mu_{N}(x)=\left\{\begin{array}{l}
L\left(\frac{m-x}{\alpha}\right), x \leq m, \alpha>0 \\
R\left(\frac{x-m}{\beta}\right), x>m, \beta>0
\end{array}\right.
$$

$\tilde{N}$ is called the fuzzy number, is denoted by. In which m is called the average

$\alpha, \beta$ known as the spread of left, right, and when $\alpha=\beta=0, L-R$ fuzzy degradation for the common real number, namely $(m ; 0,0)_{L R}=m$.

Theorem: Let $\tilde{M}=(m ; \alpha, \beta)_{L R}, \tilde{N}=(n ; \gamma, \delta)_{L R}$, Then

(1) $k \tilde{M}=(k m ; k \alpha, k \beta)_{L R}$;

(2) $\tilde{M}+\tilde{N}=(m+n ; \alpha+\gamma, \beta+\delta)_{L R}$;

(3) $\tilde{M}-\tilde{N}=(m-n ; \alpha+\gamma, \beta+\delta)_{L R}$.

Definition 2.5 Let $\tilde{A}, \tilde{B}$ be two fuzzy Numbers, $\tilde{A} \phi, \tilde{B}$ is used to denote fuzzy number $\tilde{A}$ is better than fuzzy Number $\tilde{B}, v \phi(\tilde{A}, \tilde{B})$ denotes the possibility of that fuzzy Numbers $\tilde{A}$ is better than that of fuzzy number $\tilde{B}$, which

$$
v_{\phi}(\tilde{A}, \tilde{B})=\sup _{x, y \in R, x \geq y}\left[\min \left(\mu_{\tilde{A}}(x), \mu_{\tilde{B}}(y)\right)\right](*)
$$

If $v_{\phi}(\tilde{A}, \tilde{B})>v_{\phi}(\tilde{B}, \tilde{A})$, the degree of that fuzzy number $\tilde{A}$ is better than fuzzy number $\tilde{B}$ is bigger than the degree of that fuzzy number $\tilde{B}$ is better than fuzzy number $\tilde{A}$;

If $v_{\phi}(\tilde{A}, \tilde{B})<v_{\phi}(\tilde{B}, \tilde{A})$, the degree of that fuzzy number $\tilde{A}$ is better than fuzzy number $\tilde{B}$ is smaller than the degree of that fuzzy number $\tilde{B}$ is better than fuzzy number $\tilde{A}$;

If $v_{\phi}(\tilde{A}, \tilde{B})=v_{\phi}(\tilde{B}, \tilde{A})$, fuzzy number $\tilde{A}$ is equivalent to fuzzy Numbers $\tilde{B}$.

Note: under the definition $(*)$, there must be $\max \left\{v_{\phi}(\tilde{A}, \tilde{B}), v_{\phi}(\tilde{B}, \tilde{A})\right\}=1$ (this is due to the fuzzy number is normal, convex).

The $L-R$ fuzzy Numbers are more intuitive and simple processing method, the following discussion we limited to the $L-R$ fuzzy number.

\section{The Method of Fuzzy Analysis of Risk Investment Decision}

3.1 the fuzzy analysis method of net present value when the economic life period is defined

when a certain of In investment decision making under the condition that the factors of investment decision are uncertain, it will be described with probability, the traditional analysis method through calculating some basic contents as expectation and standard deviation, replaced with the fuzzy analysis method, specific calculation method is as follows:

Let $\tilde{X}_{1}=\left(m_{1}, \alpha_{1}, \beta_{1}\right)$ be the fuzzy net cash flow of an investment plan in the $t$ phase, be expressed with $L-R$ fuzzy number; let $I_{t}$ be the amount of net cash investment in the $t$ phase $t=0,1,2, \ldots, m$, Then the net present value of net cash flow is $\tilde{R}_{p}=\sum_{t-0}^{m} \tilde{X}_{t}(1+r)^{-t}$, where $r$ is the riskfree rate.

The net present value to the total cash investment is: $I_{p}=\sum_{t=0}^{m} I_{t}(1+r)^{-t}$

Net present value rate is: $\tilde{K}_{p}=\tilde{R}_{p} / I_{p}$

We can get the fuzzy number $\tilde{R}_{p}, \tilde{K}_{p}$ through the above calculation, that is the fuzzy analysis method's basementnet of present value of risky investment decision.

Description:

(1) vague solving way is closer to people's mode of thinking, is more humanized analysis method.

(2) Decision analysis based on fuzzy order relation has a variety of methods, here we select the commonly used method given in definition 2.5 .

Therefore, when the economic life period of investment plan is defined, the steps of the fuzzy decision analysis method of net present value (rate) are as follows:

Calculate net present value $\tilde{R}_{p}$ of net cash flow of the program ;

(2) Calculate the net present value $I_{p}$ of total cash investment of each scheme;

(3) If the $I_{p}$ of each scheme are equal, using $\tilde{R}_{p}$ for selection according to the definition 5; Otherwise using fuzzy number $\tilde{K}_{p}$ to make sure the optimal solution ccording to the definition 5.

3.2 The fuzzy analysis method of the net present value (rate) when the economic life period is not defined.

If the economic life period $t=\left(t_{1}, t_{2}, \ldots, t_{q}\right)$ of investment 
plan has a probability distribution $P\left(T=t_{j}\right)=P\left(t_{j}\right)$, then

$$
\begin{aligned}
& \tilde{R}_{p}=\sum_{j=1}^{q} p\left(t_{j}\right) \sum_{t=0}^{t_{j}} \tilde{R}_{t}^{j}(1+r)^{-t} \\
& I_{p}=\sum_{j=1}^{q} p\left(t_{j}\right) \sum_{t=0}^{t_{j}} I_{t}(1+r)^{-t}
\end{aligned}
$$

The steps ofdecision analysis as above.

\section{References}

[1] Alba, E., Cotta, C. and Troya, JM. (1999). Evolutionary design of fuzzy logic controllersusing strongly-typed GP, Mathware \& soft computing, Vol. 6, No. 1, 1999, page 109-124.

[2] Anderson, T.W. (1984). An introduction to multivariate statistical analysis. Wiley, 2ndedition, New York.
[3] Altman, E.I., (1968). Financial ratios, discriminant analysis and the prediction ofcorporate bankruptcy. The Journal of Finance 23 (4), 589609.

[4] Asuncion, A. and Newman, D.J. (2007). UCI Machine Learning Repository[http://www.ics.uci.edu/ mlearn/MLRepository.html]. Irvine, CA: University ofCalifornia, Department of Information and Computer Science.

[5] Back, B et al. (1996). Choosing bankruptcy predictors using discriminant analysis, logitanalysis, and genetic algorithms, in: Proceedings ofthe 1st International Meeting onArtificial Intelligence in Accounting, Finance and Tax, pp. 337-356.

[6] Baesens, B., Gestel,T., Viane,S., Stepanova,M., Suykens,J and Vanthienen,J. (2003a).Benchmarking state-of-the-art classification algorithms for credit scoring. Journal ofthe Operational Research Society, Volume 54, Issue 5, pages 627-635.

[7] Baesens, B., Setiono, R., Mues, C., and Vanthienen, J. (2003b). Using neural networkrule extraction and decision tables for credit-risk evaluation. Management Science, 49(3):312.329. 